BMJ Open

Diabetes

Research

\& Care

\section{Inflammation increases plasma angiopoietin-like protein 4 in patients with the metabolic syndrome and type 2 diabetes}

To cite: Tjeerdema N, Georgiadi A, Jonker JT, et al. Inflammation increases plasma angiopoietin-like protein 4 in patients with the metabolic syndrome and type 2 diabetes. BMJ Open Diabetes Research and Care 2014;2:e000034. doi:10.1136/bmjdrc-2014000034

Received 13 April 2014 Revised 1 October 2014 Accepted 14 October 2014

\section{CrossMark}

For numbered affiliations see end of article.

Correspondence to Dr Nathanja Tjeerdema; N.Tjeerdema@lumc.nl

\section{ABSTRACT}

Background: Angiopoietin-like protein 4 (ANGPTL4) inhibits lipoprotein lipase and associates with dyslipidemia. The expression of ANGPTL4 is regulated by free fatty acids (FFA) that activate lipid-sensing peroxisome proliferator-activated receptors (PPARs), but FFA can also activate pattern recognition receptors including Toll-like receptor 4 (TLR4) in macrophages.

Objective: To assess whether systemic low-grade inflammation is a determinant for plasma ANGPTL4 levels in patients with the metabolic syndrome (MetS) and type 2 diabetes mellitus (T2DM).

Design: We studied 335 male participants: healthy controls (Controls), patients with the MetS without inflammation (MetS-I) and with low-grade inflammation (MetS+l), and patients with T2DM. All patients without diabetes included in the present study were initially matched for waist circumference. In plasma, ANGPTL4, C reactive protein (CRP) and metabolic parameters were determined. Underlying mechanisms were examined using human macrophages in vitro.

Results: As compared with Controls, plasma ANGPTL4 levels were increased in patients with MetS-I, MetS+I, and T2DM. Furthermore, ANGPTL4 was increased in T2DM compared with MetS-I. In fact, plasma CRP correlated positively with plasma ANGPTL4. In vitro studies showed that TLR $3 / 4$ activation largely increased the expression and release of ANGPTL4 by macrophages.

Conclusions: Plasma ANGPTL4 levels in humans are predicted by CRP, a marker of inflammation, and ANGPTL4 expression by macrophages is increased by inflammatory stimuli.

\section{INTRODUCTION}

The angiopoietin-like proteins are a family of secreted proteins that play an important role in energy metabolism. Angiopoietin-like protein 4 (ANGPTL4) is expressed in numerous cell types including adipocytes, hepatocytes, (cardio)myocytes, endothelial cells,

\section{Key messages}

- Plasma angiopoietin-like protein 4 (ANGPTL4) levels in humans are predicted by the inflammatory marker $\mathrm{C}$ reactive protein (CRP).

- Inflammatory stimuli increase ANGPTL4 expression by macrophages in vitro.

- Therefore a novel link is provided between inflammation and ANGPTL4 expression.

and macrophages. ${ }^{12}$ ANGPTL4 plays a role in plasma lipid metabolism by inhibiting the enzyme lipoprotein lipase. This action of ANGPTL4 results in suppression of the release of plasma TG-derived fatty acids and their subsequent uptake by underlying metabolic tissues including adipose tissue, skeletal muscle, and the heart, with concomitant hypertriglyceridemia. ${ }^{3-5}$

Expression of ANGPTL4 in a variety of tissues, including adipose tissue and liver, is governed by the lipid-sensing peroxisome proliferator-activated receptors (PPARs) $\alpha, \beta$, and $\gamma$, and is stimulated in vitro by free fatty acids (FFA). ${ }^{16-8}$ Accordingly, dietary modulations that increase plasma FFA levels, including prolonged fasting, very-low-calorie diet, and high-fat, high-energy diet, also increase plasma ANGPTL4 levels, most likely via activation of PPARs in tissues such as adipose tissue and liver. ${ }^{9}{ }^{10}$ Besides activating PPARs, there is compelling evidence that FFA also activate pattern recognition receptors (PRRs) in adipocytes and macrophages, including the Toll-like receptor 4 (TLR4). ${ }^{11} 12$ Accordingly, it is of interest to study the impact of inflammation and (associated) TLR activation on ANGPTL4 expression.

The metabolic syndrome (MetS) is a progressive inflammatory disease, ranging from mild dyslipidemia and impaired fasting glucose levels to full blown type 2 diabetes 
mellitus (T2DM). ${ }^{13}$ Interestingly, the progression of MetS towards T2DM is accompanied by progressive inflammation, which may result in increased plasma levels of ANGPTL4 that, in turn, could aggravate dyslipidemia. Therefore, the aim of the present study was to assess whether systemic low-grade inflammation is a determinant for plasma ANGPTL4 levels in patients with the MetS and T2DM. To get more insight into the causal relationship between inflammation and ANGPTL4 expression we assessed whether inflammatory stimuli increase ANGPTL4 expression in vitro.

\section{RESEARCH DESIGN AND METHODS \\ Human studies \\ Subjects}

To assess the effects of low-grade systemic inflammation on plasma ANGPTL4 levels we studied four groups of human participants: healthy controls (Controls, $n=95$ ), patients with MetS but without systemic inflammation (MetS-I, n=106), patients with MetS accompanied by low-grade systemic inflammation $(\mathrm{MetS}+\mathrm{I}, \mathrm{n}=66)$, and patients with T2DM $(n=68)$. All patients without diabetes included in the present study were initially matched for waist circumference (maximum difference $17 \mathrm{~cm}$ ) and age (maximum difference 19 years). The MetS was defined according to the International Diabetes Federation (IDF) criteria, ${ }^{15}$ that is, two or more of the following criteria present in addition to increased waist circumference (male $\geq 94$ ): triglycerides $\geq 1.7 \mathrm{mmol} / \mathrm{L} \quad(\geq 150 \mathrm{mg} / \mathrm{dL}) /$ the use of lipid-lowering medication, high-density lipoprotein (HDL) cholesterol $<1.03 \mathrm{mmol} / \mathrm{L} \quad(<40 \mathrm{mg} / \mathrm{dL})$ in men and $<1.29 \mathrm{mmol} / \mathrm{L}(<50 \mathrm{mg} / \mathrm{dL})$ in women, fasting glucose $\geq 5.6 \mathrm{mmol} / \mathrm{L} \quad(100 \mathrm{mg} / \mathrm{dL})$ or blood pressure (BP) $\geq 130 / \geq 85 \mathrm{~mm} \mathrm{Hg} /$ the use of BP-lowering medication. Low-grade inflammation was defined as moderately elevated $\mathrm{C}$ reactive protein $(\mathrm{CRP}): 3 \mathrm{mg} / \mathrm{L} \leq \mathrm{CRP}<15 \mathrm{mg} / \mathrm{L}$.

Controls $(n=95)$, patients with MetS-I $(n=66 ; 62 \%$ of all included patients with MetS-I) and patients with MetS+I (n=33; $50 \%$ of all included patients with MetS+I) were selected from a survey in the general population in the city of Rijswijk, the Netherlands. For this survey, all patients without diabetes between 40 and 70 years of age of four general practitioners were invited for a cardiovascular risk screening $(n=2942)$. Patients with diagnosed diabetes, known terminal disease, and a history of psychiatric disorders or substance abuse were excluded. Screening was performed on 2079 patients (response rate of $71 \%$ ) and CRP was determined for 1515 patients. After excluding patients with high CRP (defined as $>15 \mathrm{mg} / \mathrm{L}, \mathrm{n}=45)$, patients with missing values $(\mathrm{n}=13)$, female patients $(n=792)$ and patients with cardiovascular disease $(n=55)$, and 610 male patients were eligible. Only male patients were selected to avoid the potential interference of sex steroid hormones on study parameters. The MetS was present in $44 \%(n=268)$ of the 610 male patients. A total of 98 Controls and 102 patients with MetS were selected for the current study.
In addition 1 patient with new diagnosed diabetes, 1 patient with a very high level of ANGPTL4 and 4 patients with missing data ( $n=3$ Control and $n=1$ MetS) were excluded therefore leading to 95 Controls and 99 patients with MetS. Patients with MetS were divided into MetS-I $(n=66)$ and MetS+I $(n=33)$.

Patients with MetS-I $(n=40)$ and MetS+I $(n=33)$ were also selected from a previously published study on the effect of lifestyle in combination with rosiglitazone versus placebo on atherosclerosis in patients with MetS $\left(\right.$ RUBENS study $\left.{ }^{16}\right)$. These patients $(n=73)$ were matched on waist and age with the patients from the PIRAMID study (see below). For this study male patients with increased waist circumference $(\geq 94 \mathrm{~cm})$ and elevated CRP levels $(\geq 1.8 \mathrm{mg} / \mathrm{L})$ and two other MetS criteria were included. Exclusion criteria for this study were T2DM (fasting blood glucose $\geq 7 \mathrm{mmol} / \mathrm{L}$ ), manifest cardiovascular disease, use of statins, steroids or nonsteroidal anti-inflammatory drugs at baseline, heart failure, QTc time interval of $450 \mathrm{~ms}$ or longer on baseline ECG, primary dyslipidemias, presence of potential hepatic disease (ie, patients with alanine aminotransferase, total bilirubin,or alkaline phosphatase levels exceeding 2.5 times the upper limit of the normal laboratory values), alcohol abuse (>30 units/week) and cardiovascular MR contraindications.

Patients with T2DM $(n=68)$ were selected from a recently published study on the effects of pioglitazone versus placebo on cardiovascular parameters in uncomplicated T2DM (PIRAMID) study. For detailed inclusion criteria, see ref. 17 . In short, the study included male patients with T2DM without cardiovascular disease or diabetes-related complications, body mass index (BMI) 25-32 kg/m², age 45-65 years, and well-controlled T2DM with oral antidiabetic medication (glycated hemoglobin (HbA1c) 6.5-8.5\%). A total of 73 patients from the PIRAMID were matched on waist circumference and age with 73 patients from the RUBENS. A total of five patients were excluded due to CRP levels $>15(n=4)$ and missing data $(n=1)$.

The studies were executed in accordance with the principles of the Declaration of Helsinki and were approved by the local medical ethics committee. All volunteers in these studies signed an informed consent.

\section{Analysis of CRP and ANGPTL4}

CRP was determined by the Tina Quant $\mathrm{C}$ reactive protein (latex) high-sensitive assay according to the manufacturer's instructions (Roche, Basel, Switzerland). ANGPTL4 was measured by ELISA as detailed previously. ${ }^{9}$ Briefly, 96-well plates were coated with antihuman ANGPTL4 polyclonal goat IgG antibody (AF3485, R\&D Systems) and incubated overnight at $4^{\circ} \mathrm{C}$. Plates were washed extensively between each step. After blocking, $100 \mu \mathrm{L}$ of undiluted medium or 20 -fold diluted human plasma was added, followed by $2 \mathrm{~h}$ incubation at room temperature. A standard curve was prepared using recombinant human ANGPTL4 (3485-AN, R\&D 
Systems) at $0.3-2.1 \mathrm{ng} /$ well. Next, $100 \mu \mathrm{L}$ of diluted biotinylated antihuman ANGPTL4 polyclonal goat IgG antibody (BAF3485, R\&D Systems) was added for $2 \mathrm{~h}$, followed by addition of streptavidin-conjugated horseradish peroxidase for $20 \mathrm{~min}$, and tetramethyl benzidine substrate reagent for $6 \mathrm{~min}$. The reaction was stopped by addition of $50 \mu \mathrm{L}$ of $10 \% \mathrm{H}_{2} \mathrm{SO}_{4}$, and the absorbance was measured at $450 \mathrm{~nm}$.

\section{In vitro studies}

Cell culture

Human THP-1 and U937 monocytes were grown in RPMI medium containing $10 \%$ heat-inactivated fetal calf serum (FCS) and 1\% $\mathrm{P} / \mathrm{S}$ for 10-15 passages. They were differentiated into macrophages after 2 days of incubation by adding $100 \mathrm{ng} / \mathrm{mL}$ phorbol-12-myristate-13-acetate (PMA). After 2 days, PMA was removed by washing with phosphate-buffered saline and macrophages were kept in complete medium without PMA for two additional days and then used for experiments. Human peripheral blood mononuclear cells (PBMCs) were isolated from whole blood by using BD Vacutainer Cell Preparation Tubes according to the manufacturer's instructions. Isolated PBMCs were plated in complete medium RPMI, $10 \%$ heat-inactivated FCS, $1 \% \mathrm{P} / \mathrm{S}$. After $2 \mathrm{~h}$ cells were washed and remaining attached cells were differentiated into macrophages in completed medium, containing $100 \mathrm{ng} / \mathrm{mL}$ macrophage colony stimulating factor, for five subsequent days. During differentiation medium was not refreshed.

\section{Incubations}

THP-1 cells were incubated with the TLR agonists (Sigma, Fluka, Brunwich, and InvivoGen) lipopolysaccharide (LPS, TLR4; $100 \mathrm{ng} / \mathrm{mL}$ or $1 \mu \mathrm{g} / \mathrm{mL}$ ), $S$ (2,3-bispalmitoyloxypropyl)-Cys-Gly-Asp-Pro-Lys-His-ProLys-Ser-Phe, Pam2CGDPKHPKSF (fibroblast-stimulating lipopeptide; FSL-1, TLR2, and TLR6; $1 \mu \mathrm{g} / \mathrm{mL})$, tri-acylated lipopeptide Pam3-Cys-Ser-Lys4 (Pam3, TLR1 and TLR2; $1 \mu \mathrm{g} / \mathrm{mL}$ ), polyinosinic-polycytidylic acid (poly (I:C), TLR3; $2 \mu \mathrm{g} / \mathrm{mL}$ ), muramyl dipeptide (MDP, NOD2; $10 \mu \mathrm{g} / \mathrm{mL}$ ), flagellin (Flag, TLR5; $10 \mathrm{ng} / \mathrm{mL}$ ) and/or FFA-donor intralipid ( $2 \mathrm{mM}$; Fresenius Kabi) for the indicated time periods at $37^{\circ} \mathrm{C}$. After incubation, medium was aspired and cells were washed. Cellular ANGPTL4 mRNA was determined by qPCR (forward primer CACAGCCT GCAGACACAACTC; reverse primer GGAGGCCAAACT GGCTTTGC) and ANGPTL4 protein in the medium was determined by ELISA as detailed above.

\section{Statistical analysis}

Data are expressed as mean $\pm \mathrm{SD}$ or as median (IQR) if the assumption of normality was not met. Categorical data are presented as frequencies (\%). Comparisons between continuous variables were assessed by independent $t$ test or one-way analysis of variance (ANOVA), followed by Bonferroni's post hoc test for multiple comparisons (table 1 and figure 1). Comparisons between categorical data were performed with $\chi^{2}$ tests (with the level of significance adjusted for the number of comparisons, table 1). The correlation between clinical characteristics and ANGPTL4 was tested by Spearman's correlation coefficients (figure 2) and multivariable regression analysis with logarithmically transformed ANGPTL4 values. Statistical analysis was performed using SPSS for Windows (V.17.0; SPSS, Chicago, Illinois, USA). Plots were created with GraphPad Prism V.5.0 (GraphPad Software, Inc, La Jolla, California, USA). p Value $<0.05$ was considered statistically significant.

\section{RESULTS}

\section{Characteristics of the human study population}

To evaluate whether low-grade inflammation is associated with plasma ANGPTL4 levels in humans, we compared Controls $(\mathrm{n}=95)$ with patients with MetS-I $(\mathrm{n}=106)$, MetS+I $(\mathrm{n}=66)$ and T2DM $(\mathrm{n}=68)$.

The mean age of all male patients combined $(n=335)$ was $56.9 \pm 6.0$ years and mean waist circumference was 105 $\pm 10 \mathrm{~cm}$. Clinical characteristics of the study population are described in table 1. Groups matched for age and waist circumference did not differ with regard to FFA and family history of cardiovascular disease. Fasting glucose and triglyceride levels were higher, and HDL-cholesterol levels were lower in MetS-I, MetS+I and T2DM compared with Controls. BP was higher in MetS-I and MetS +I compared with Controls and T2DM. Patients with T2DM used more statins and had lower total cholesterol compared with MetS-I, MetS+I and Controls. Furthermore patients with MetS+I smoked more frequently then Controls and patients with T2DM. Low-grade inflammation measured as CRP was highest in MetS+I (5.55 (4.11-8.02) mg/L, $\mathrm{p}<0.001$ compared with all groups), followed by T2DM (3.60 (2.43-5.95) $\mathrm{mg} / \mathrm{L})$, MetS-I (1.96 (0.92-2.37) mg/L) and Controls (1.43 (0.76-2.85) $\mathrm{mg} / \mathrm{L})$.

\section{Plasma ANGPTL4 levels}

The median plasma ANGPTL4 in the total study population was $5.00(3.50-6.80) \mathrm{ng} / \mathrm{mL}$ and ranged from 0.90 to $43.30 \mathrm{ng} / \mathrm{mL}$. The distribution of ANGPTL4 was skewed to the right. Figure 1 shows the plasma ANGPTL4 levels in Controls, MetS-I, MetS+I and T2DM. Plasma ANGPTL4 was higher in T2DM $(6.4(5.2-8.2) \mathrm{ng} / \mathrm{mL}$, $\mathrm{p}<0.001)$, in MetS+I $(6.0(3.8-7.7) \mathrm{ng} / \mathrm{mL}, \mathrm{p}<0.001)$ and MetS-I (4.6 (3.3-6.5) ng/mL, p=0.006), than in Controls (3.7 (2.8-5.2) ng/mL). In addition, higher ANGPTL4 was found in T2DM compared with MetS-I $(p<0.001$; table 1).

\section{Relationship between plasma ANGPTL4, metabolic parameters and low-grade inflammation}

Although groups were matched for waist circumference, in all patients combined plasma ANGPTL4 positively correlated with waist circumference $(\mathrm{r}=0.195, \mathrm{p}<0.001)$ and fasting glucose $\quad(\mathrm{r}=0.322, \quad \mathrm{p}<0.001) . \quad$ Plasma 


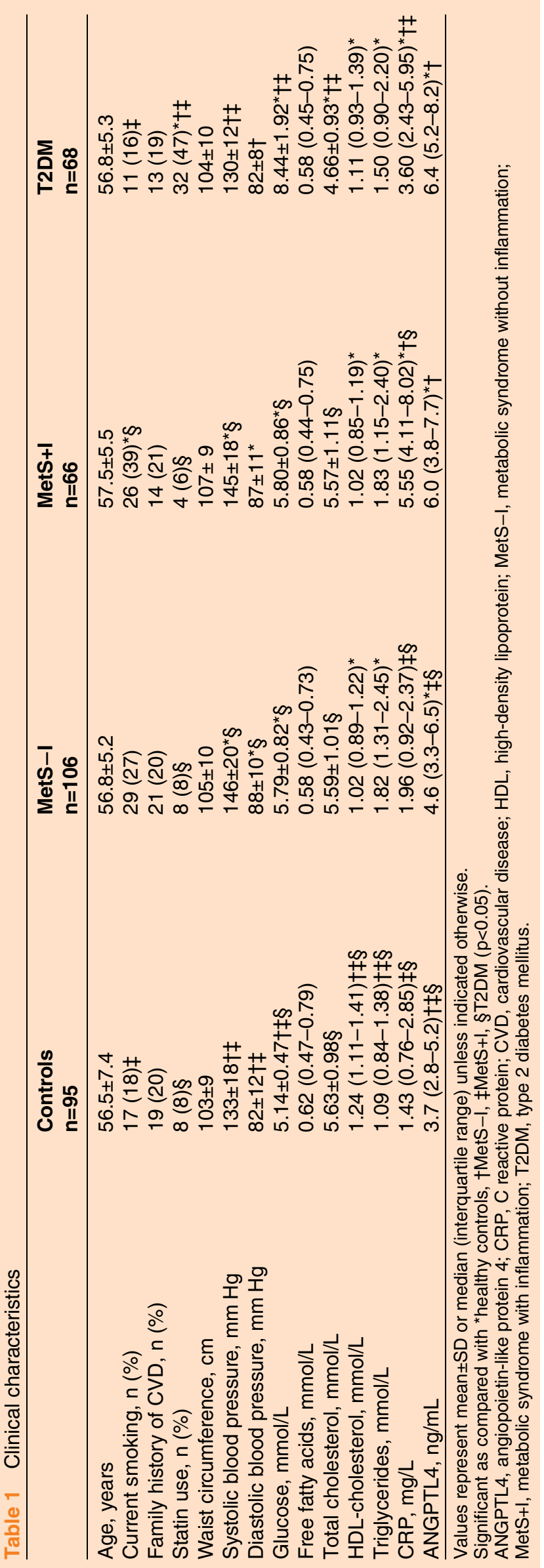

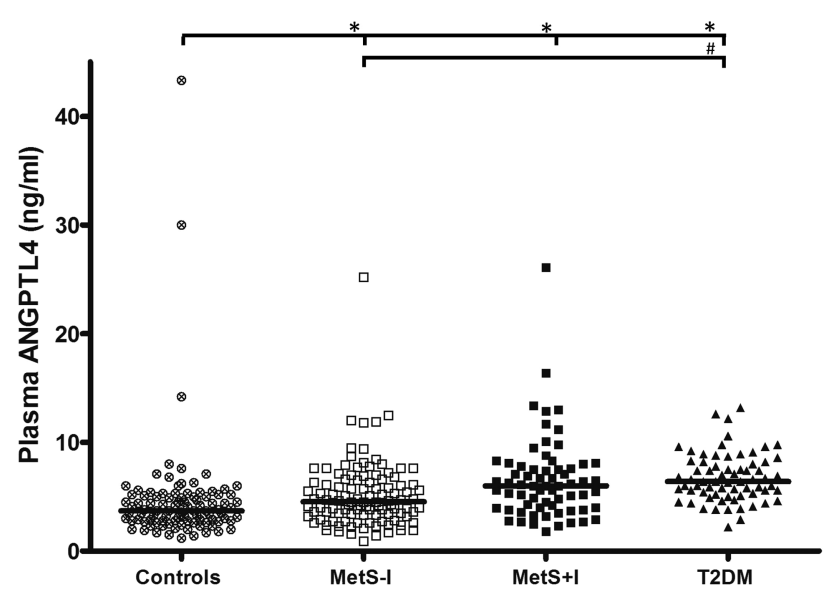

Figure 1 Plasma angiopoietin-like protein 4 (ANGPTL4) in healthy controls (Controls), patients with metabolic syndrome without (MetS-I) and with low-grade inflammation (MetS+l) and patients with type 2 diabetes mellitus (T2DM). Plasma was taken from Controls, patients with MetS-I, MetS + I and T2DM, and plasma ANGPTL4 levels were determined. As compared with plasma ANGPTL4 levels (median (IQR)) in Controls (3.7 (2.8-5.2) ng/mL), ANGPTL4 levels are increased in patients with MetS-I (4.6 (3.3-6.5) ng/mL, $\mathrm{p}=0.006)$, patients with MetS+l $(6.0(3.8-7.7) \mathrm{ng} / \mathrm{mL}, \mathrm{p}<0.001)$ and patients with T2DM $(6.4$ (5.2-8.2) ng/mL, $\mathrm{p}<0.001)$. Furthermore, ANGPTL4 was increased in T2DM compared with MetS-I $(p<0.001)$. Significant as compared with Controls. ${ }^{*} p<0.001, \# p<0.05$.

ANGPTL4 negatively correlated with HDL-cholesterol $(\mathrm{r}=-0.143, \mathrm{p}=0.009)$ and total cholesterol $(\mathrm{r}=-0.223$, $\mathrm{p}<0.001)$. Plasma ANGPTL4 did not correlate with age $(\mathrm{p}=0.457)$, triglycerides $(\mathrm{p}=0.055)$ or FFA $(\mathrm{p}=0.226)$.

Plasma ANGPTL4 showed a positive correlation with CRP ( $\mathrm{r}=0.295, \mathrm{p}<0.001$; figure 2). Multiple regression analysis revealed that the association between ANGPTL4 and CRP was independent of age, waist circumference, glucose, HDL-cholesterol, triglycerides, total cholesterol, FFA, and smoking $(\beta=0.142, p=0.010)$.

\section{Inflammation-dependent ANGPTL4 expression in human macrophages}

Since the above described correlations do not necessarily implicate causal relationships, we performed mechanistic studies in vitro to get more insight into potential causality between inflammation and ANGPTL4 expression. To demonstrate that ANGPTL4 expression is sensitive to stimulation by inflammatory stimuli, we treated macrophages with various PRR agonists and determined ANGPTL4 expression (figure 3). We used human THP-1 macrophages because: (1) they are human macrophages, (2) they secrete sufficient amounts of ANGPTL4 to allow quantification in the cell medium.

In human THP-1 macrophages ANGPTL4 mRNA expression (figure 3A) and ANGPTL4 protein secretion (figure 3B) were strongly increased by the TLR4 agonist LPS and the TLR3 agonist poly(I:C). Consistent with 


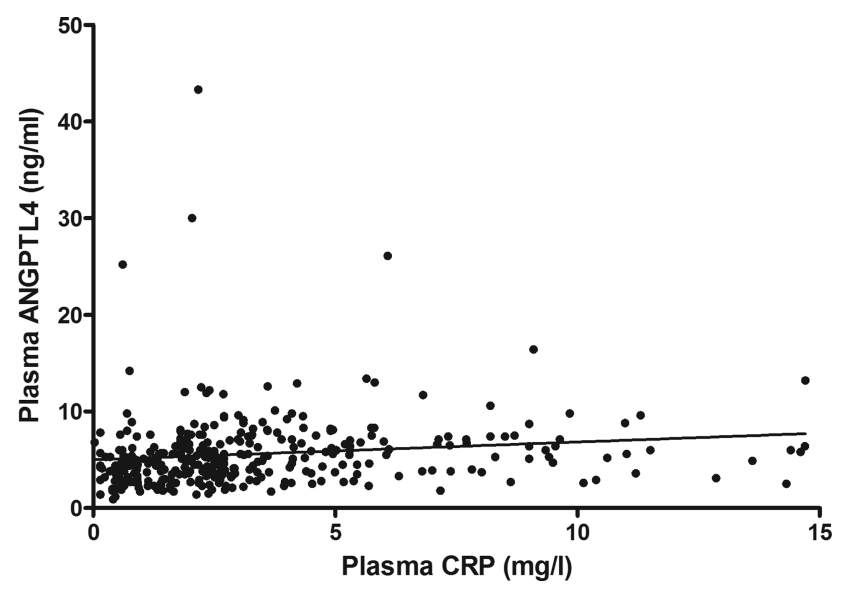

Figure 2 Correlation between plasma $C$ reactive protein (CRP) and angiopoietin-like protein 4 (ANGPTL4). Plasma was taken from healthy controls, patients with metabolic syndrome without and with low-grade inflammation and patients with type 2 diabetes mellitus, and plasma ANGPTL4 levels as well as CRP levels were determined. Plasma CRP correlates positively with plasma ANGPTL4 ( $r=0.295$, $\mathrm{p}<0.001)$.

these data, LPS also increased ANGPTL4 mRNA expression in human U937 macrophages and human monocyte-derived macrophages (figure 3C). Intralipid, a source of FFA that induces foam cell formation, also increased ANGPTL4 mRNA (figure 3D) and ANGPTL4 protein (figure 3E) expression in THP-1 macrophages, but no clear synergy was observed between Intralipid and LPS towards induction of ANGPTL4. Taken together, these data show that inflammatory stimuli causing macrophage activation and macrophage foam cell formation markedly increase ANGPTL4 expression in human macrophages.

\section{DISCUSSION}

The main findings of our cross-sectional human study are: (1) patients with MetS and T2DM show increased plasma ANGPTL4 levels compared with Controls, (2) patients with MetS+I show higher levels of ANGPTL4 compared with patients with MetS-I, (3) plasma CRP, as a marker of low-grade inflammation, positively correlates with plasma ANGPTL4, independent of age, waist circumference, glucose and lipid variables.

Thus far, the expression of ANGPTL4 in numerous tissues including adipose tissue and liver was thought to be mainly regulated by PPARs. Consistent with a role of PPARs, fatty acids increase ANGPTL4 expression in vitro in numerous cell types. ${ }^{16-8}$ Furthermore, in vivo modulation of plasma FFA levels also modulates plasma ANGPTL4 levels, ${ }^{9}{ }^{10}$ with a positive correlation observed between the change in FFA levels and change in ANGPTL4 levels. ${ }^{10}$ In our previous study, we postulated that patients with T2DM may have higher ANGPTL4 levels than healthy participants due to elevated plasma FFA. ${ }^{10}$ Although in the present paper we do observe increased plasma ANGPTL4 levels in patients with T2DM compared with Controls, plasma FFA levels were not different between both groups. In addition, plasma ANGPTL4 levels were elevated in patients with MetS compared with Controls, whereas their plasma FFA levels did not differ from the healthy Controls. In fact, in this cross-sectional study we were unable to detect a correlation between plasma FFA levels and plasma ANGPTL4 levels, which is likely related to the matching on visceral obesity and the subsequent limited variation in FFA between groups.

Therefore, we hypothesized that additional factors such as inflammation may contribute to the increased ANGPTL4 levels in patients with MetS and T2DM. Indeed, we did observe a progressive increase in the plasma ANGPTL4 in males according to inflammatory status (healthy Controls < patients with MetS-I < patients with MetS+I and patients with T2DM). Furthermore, in all groups combined, plasma ANGPTL4 showed a positive correlation with CRP, an established marker for low-grade inflammation.

To get more insight into the causal relationship between inflammation and ANGPTL4 expression we treated human macrophages with various PRR ligands. Macrophages were used because of their sensitivity to inflammatory stimuli, not because they are suspected to be solely responsible for the changes in plasma ANGPTL4 levels observed in vivo. Of all ligands tested, agonists for TLR3 (ie, poly(I:C)) and TLR4 (ie, LPS) consistently induced the expression and release of ANGPTL4 in different types of human macrophages. It remains unclear which intracellular pathway activated by TLR activation is responsible for induction of ANGPTL4. Also, it is unsure whether FFA, known to activate PRRs such as TLR4 in adipocytes and macrophages, ${ }^{11} 12$ may increase ANGPTL4 expression via TLR4 activation. It should be noted that the similarity in plasma FFA levels between the various groups despite major differences in plasma ANGPTL4 levels, suggests that plasma FFA are not a major determinant of ANGPTL4 in the population studied. Since CRP itself is a marker rather than a mediator of inflammation, ${ }^{18}$ the nature of the inflammatory stimuli that cause an increase in plasma ANGPTL4 in humans evidently still has to be identified. Also, it would be of interest to study the response of ANGPTL4 to inflammatory stimuli in other cell types, including human adipocytes.

We hypothesized that the inflammation induced increase in plasma ANGPTL4 in patient with MetS and T2DM may induce further dyslipidemia. Indeed, taken all groups together, we observed a modest negative correlation between ANGPTL4 and plasma HDL-cholesterol $(\mathrm{r}=-0.143 ; \mathrm{p}<0.009)$. Some limitations do apply to our study. First of all, we previously showed that the ANGPTL4 ELISA measures full-length ANGPTL4 and the C-terminal truncated fragment of ANGPTL4 but not the N-terminal truncated fragment. Only full-length and the N-terminal fragment of ANGPTL4 influence plasma lipids. It is unclear to what 
A

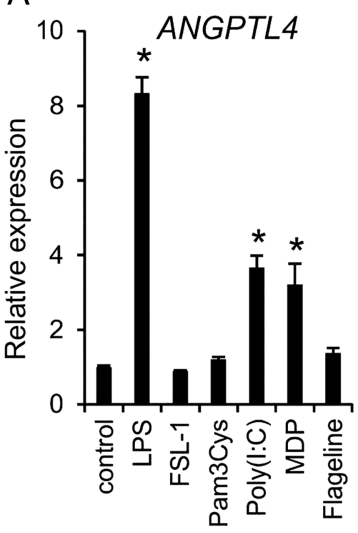

C

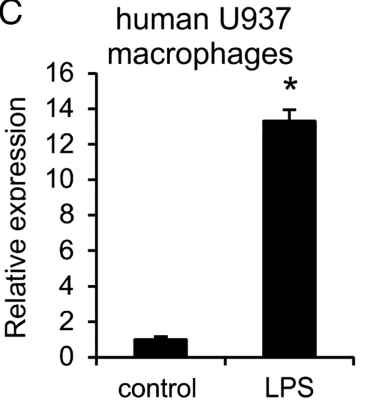

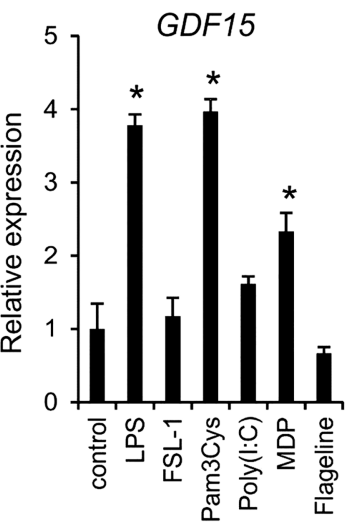

human monocyte

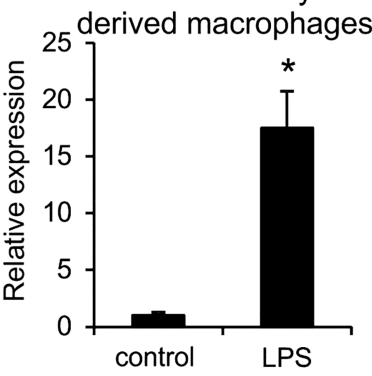

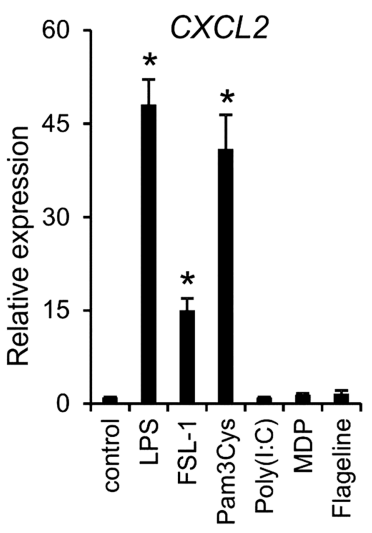

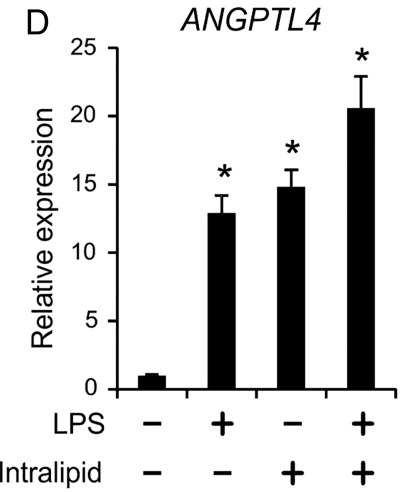

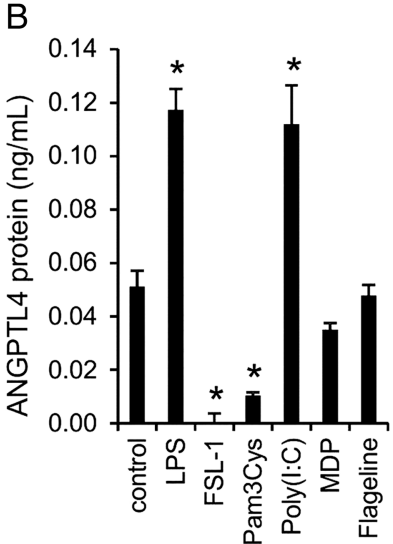

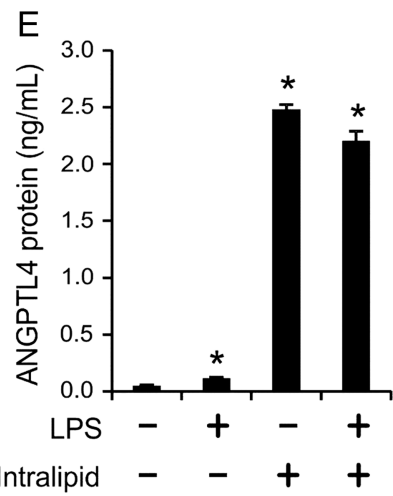

Figure 3 Inflammatory stimuli increase angiopoietin-like protein 4 (ANGPTL4) gene and protein expression in human macrophages. (A) Human THP-1 macrophages were incubated with various Toll-like receptor (TLR) agonists for $12 \mathrm{~h}$, and changes in expression of ANGPTL4, Gdf15 and Cxcl2 were determined. (B) Human THP-1 macrophages were incubated with various TLR agonists for $24 \mathrm{~h}$, and ANGPTL4 levels in medium were assessed. (C) Human U937 macrophages were incubated with lipopolysaccharide (LPS) (100 ng/mL; $4 \mathrm{~h}$ ) and ANGPTL4 mRNA expression was determined; human monocyte-derived macrophages were incubated with LPS $(1 \mu \mathrm{g} / \mathrm{mL} ; 24 \mathrm{~h})$ and ANGPTL4 mRNA expression was determined. (D and E) THP-1 macrophages were incubated with LPS, intralipid, or both for $24 \mathrm{~h}$ and ANGPTL4 mRNA expression (D) or protein secretion (E) were determined. Values are means \pm SEM $(n=3-6)$. Differences as compared with controls were evaluated by Student $t$ test. ${ }^{*} \mathrm{p}<0.05$.

extent the plasma concentrations of C-terminal and fulllength ANGPTL4 reflect the concentration of N-terminal ANGPTL4. The mechanism underlying the relationship between inflammation and plasma ANGPTL4 levels is unknown as yet, and probably involves causal stimuli other than CRP that merely represents a marker of inflammation. Further studies into the causal relationships are thus required. Also, it is unclear why CRP is lower in T2DM compared with patients with MetS+I. It should be noted that patients with wellcontrolled diabetes with an HbAlc between $6.5 \%$ and $8.5 \%$, a BMI between $25-32 \mathrm{~kg} / \mathrm{m}^{2}, \mathrm{BP}<150 / 85 \mathrm{~mm} \mathrm{Hg}$, and no history of diabetes-related complications were included, who were treated with drugs such as statins with pleotropic anti-inflammatory effects. Furthermore, higher CRP in patients with MetS+I may be explained by a higher percentage of smokers in that group. Also, from the current study set up it is unclear whether the relationship between ANGPTL4 and CRP in humans is solely explained by activation of macrophages. Other cell types and tissues may also be involved.
In conclusion, we provide a novel link between inflammation and ANGPTL4. An increased inflammatory state in patients with MetS and T2DM was associated with higher plasma ANGPTL4 levels. Additionally, a positive correlation was observed between plasma CRP and ANGPTL4. Although in vitro studies confirmed that PRR ligands increase ANGPTL4 expression, further studies into the precise mechanisms underlying the relationship between inflammation and ANGPTL4 in humans are warranted.

\section{Author affiliations}

${ }^{1}$ Departments of Endocrinology and Metabolic Diseases, Leiden University Medical Center, Leiden, The Netherlands

${ }^{2}$ Nutrition, Metabolism and Genomics group, Wageningen University, Wageningen, The Netherlands

${ }^{3}$ Department of Internal Medicine, University Medical Center Nijmegen, Nijmegen, The Netherlands

${ }^{4}$ Department of Einthoven Laboratory for Experimental Vascular Medicine, Leiden University Medical Center, Leiden, The Netherlands

Acknowledgements The authors acknowledge the support from "the Netherlands CardioVascular Research Initiative: the Dutch Heart Foundation, 
Dutch Federation of University Medical Centers, the Netherlands Organization for Health Research and Development and the Royal Netherlands Academy of Sciences" for the GENIUS project "Generating the best evidence-based pharmaceutical targets for atherosclerosis" (CVON2011-19).

Contributors NT researched data, contributed to discussion and wrote the manuscript. AG researched data and wrote the manuscript. JTJ researched data, contributed to discussion and reviewed/edited the manuscript. MvG and RAD researched data and contributed to discussion. JTT contributed to discussion. JWAS reviewed/edited the manuscript. SK contributed to discussion and reviewed/edited manuscript. PCNR wrote and reviewed/edited the manuscript.

Funding This research was performed within the framework of CTMM, the Center for Translational Molecular Medicine (http://www.ctmm.nl), project PREDICCt (grant 01C-104) and supported by the Netherlands Heart Foundation, Dutch Diabetes Research Foundation, and Dutch Kidney Foundation. PCNR is Established Investigator of the Netherlands Heart Foundation (grant 2009T038).

Competing interests None.

Ethics approval Local medical ethics committee.

Provenance and peer review Not commissioned; externally peer reviewed.

Data sharing statement No additional data are available.

Open Access This is an Open Access article distributed in accordance with the Creative Commons Attribution Non Commercial (CC BY-NC 4.0) license, which permits others to distribute, remix, adapt, build upon this work noncommercially, and license their derivative works on different terms, provided the original work is properly cited and the use is non-commercial. See: http:// creativecommons.org/licenses/by-nc/4.0/

\section{REFERENCES}

1. Kersten S, Mandard S, Tan NS, et al. Characterization of the fasting-induced adipose factor FIAF, a novel peroxisome proliferator-activated receptor target gene. J Biol Chem 2000;275:28488-93.

2. Yoon JC, Chickering TW, Rosen ED, et al. Peroxisome proliferator-activated receptor gamma target gene encoding a novel angiopoietin-related protein associated with adipose differentiation. Mol Cell Biol 2000;20:5343-9.

3. Lichtenstein L, Berbee JF, van Dijk SJ, et al. Angpt/4 upregulates cholesterol synthesis in liver via inhibition of LPL- and HL-dependent hepatic cholesterol uptake. Arterioscler Thromb Vasc Biol 2007;27:2420-7.

4. Sukonina V, Lookene A, Olivecrona T, et al. Angiopoietin-like protein 4 converts lipoprotein lipase to inactive monomers and modulates lipase activity in adipose tissue. Proc Natl Acad Sci USA 2006;103:17450-5.

5. Yoshida K, Shimizugawa T, Ono M, et al. Angiopoietin-like protein 4 is a potent hyperlipidemia-inducing factor in mice and inhibitor of lipoprotein lipase. J Lipid Res 2002;43:1770-2.

6. Kaddatz K, Adhikary T, Finkernagel F, et al. Transcriptional profiling identifies functional interactions of TGF beta and PPAR beta/delta signaling: synergistic induction of ANGPTL4 transcription. J Biol Chem 2010;285:29469-79.

7. Lichtenstein L, Mattijssen $\mathrm{F}$, de Wit NJ, et al. Angptl4 protects against severe proinflammatory effects of saturated fat by inhibiting fatty acid uptake into mesenteric lymph node macrophages. Cell Metab 2010;12:580-92.

8. Mandard S, Zandbergen F, Tan NS, et al. The direct peroxisome proliferator-activated receptor target fasting-induced adipose factor (FIAF/PGAR/ANGPTL4) is present in blood plasma as a truncated protein that is increased by fenofibrate treatment. $\mathrm{J}$ Biol Chem 2004;279:34411-20.

9. Kersten S, Lichtenstein L, Steenbergen E, et al. Caloric restriction and exercise increase plasma ANGPTL4 levels in humans via elevated free fatty acids. Arterioscler Thromb Vasc Biol 2009;29:969-74.

10. Jonker JT, Smit JW, Hammer S, et al. Dietary modulation of plasma angiopoietin-like protein 4 concentrations in healthy volunteers and in patients with type 2 diabetes. Am J Clin Nutr 2013;97:255-60.

11. Holland WL, Bikman BT, Wang LP, et al. Lipid-induced insulin resistance mediated by the proinflammatory receptor TLR4 requires saturated fatty acid-induced ceramide biosynthesis in mice. $J$ Clin Invest 2011;121:1858-70.

12. Shi $\mathrm{H}$, Kokoeva MV, Inouye $\mathrm{K}$, et al. TLR4 links innate immunity and fatty acid-induced insulin resistance. J Clin Invest, 2006;116:3015-25.

13. Shoelson SE, Lee J, Goldfine AB. Inflammation and insulin resistance. J Clin Invest 2006;116:1793-801.

14. Velloso LA, Eizirik DL, Cnop M. Type 2 diabetes mellitus-an autoimmune disease? Nat Rev Endocrinol 2013;9:750-5.

15. Alberti KG, Zimmet P, Shaw J. The metabolic syndrome-a new worldwide definition. Lancet 2005;366:1059-62.

16. Roes SD, Dehnavi RA, Westenberg JJ, et al. Effect of lifestyle intervention plus rosiglitazone or placebo therapy on left ventricular mass assessed with cardiovascular magnetic resonance in the metabolic syndrome. J Cardiovasc Magn Reson 2011;13:65.

17. van der Meer RW, Rijzewijk LJ, de Jong HW, et al. Pioglitazone improves cardiac function and alters myocardial substrate metabolism without affecting cardiac triglyceride accumulation and high-energy phosphate metabolism in patients with well-controlled type 2 diabetes mellitus. Circulation 2009;119: 2069-77.

18. Pearson TA, Mensah GA, Alexander RW, et al. Markers of inflammation and cardiovascular disease: application to clinical and public health practice: a statement for healthcare professionals from the Centers for Disease Control and Prevention and the American Heart Association. Circulation 2003;107:499-511. 